\title{
Études cinétiques de la dismutation du monoxyde d'étain SnO
}

\author{
C. Machado-Bailly, H. Delalu, J.M. Létoffé et R. Metz \\ Laboratoire Hydrazines et Procédés, UMR 5179 UCBL-CNRS-Groupe SNPE, \\ Université Claude Bernard Lyon I, 69622 Villeurbanne Cedex, France
}

\begin{abstract}
Résumé : Ce travail est basé sur la préparation, par un nouveau procédé (ODAP : Oxydation Directe d'un Alliage Précurseur) de poudres céramiques $\mathrm{SnO}_{2}$. Une des étapes importantes du procédé étant la conversion totale du métal en l'oxyde correspondant, l'oxydation d'étain en dioxyde d'étain a été étudiée. Cette oxydation s'est révélée complexe, puisqu'elle fait intervenir le monoxyde d'étain $\mathrm{SnO}$. Or cet oxyde intermédiaire subit une réaction de dismutation donnant lieu à la formation des phases $\mathrm{Sn}, \mathrm{Sn}_{3} \mathrm{O}_{4}$ et $\mathrm{SnO}_{2}$ selon les températures de traitement. Cette dismutation a été suivie par diffraction des rayons $\mathrm{X}$ : les produits de réaction ont été identifiés et les cinétiques de formation étudiées. Pour des températures de 400 et $480^{\circ} \mathrm{C}$, la dismutation a lieu en deux temps : décomposition rapide de $\mathrm{SnO}$ en $\mathrm{Sn}$ et $\mathrm{Sn}_{3} \mathrm{O}_{4}$ puis dégradation plus lente de $\mathrm{Sn}_{3} \mathrm{O}_{4}$ en $\mathrm{Sn}$ et $\mathrm{SnO}_{2}$. Une étude morphologique a ensuite montré l'hétérogénéité des échantillons ayant subi la dismutation et notamment la présence de billes solides pouvant être attribuées à de l'étain liquide solidifié.
\end{abstract}

\section{INTRODUCTION}

Les céramiques à base de dioxyde d'étain sont de nouveaux matériaux utilisés dans de nombreux domaines tels que les détecteurs de gaz $[1,2]$. Elles sont généralement élaborées par le procédé traditionnel d'élaboration des céramiques, à savoir le mélange-broyage d'oxydes simples.

Le laboratoire a mis au point un procédé original d'élaboration de céramiques : le procédé ODAP (Oxydation Directe d'un Alliage Précurseur) [3]. Il consiste tout d'abord en l'élaboration d'un alliage liquide contenant les éléments constitutifs de la future céramique, puis en l'atomisation et trempe de cet alliage de façon à le diviser en fines particules. La poudre métallique obtenue est alors oxydée. La poudre céramique résultante est enfin mise en forme et frittée pour aboutir à un matériau massif dense.

Afin de contrôler l'oxydation des poudres d'alliage (contenant une majorité d'étain), une étude a été menée sur l'oxydation de poudres métalliques d'étain pur. Or, cette oxydation n'est pas simple car elle fait intervenir l'oxyde intermédiaire $\mathrm{SnO}$, oxyde connu pour sa dismutation $[4,5]$.

L'objectif est donc d'étudier cet oxyde intermédiaire $\mathrm{SnO}$, et en particulier sa dismutation et son oxydation pour ensuite identifier son influence dans l'oxydation de l'étain.

\section{CONDITIONS EXPERIMENTALES}

La poudre de monoxyde d'étain $\mathrm{SnO}$ utilisée pour cette étude est de marque Alfa Aesar. Sa pureté est de $99,9 \%$ et l'analyse en Microscopie Electronique à Balayage a montré qu'elle se compose de parallélépipèdes de largeur et longueur de l'ordre de 20 à $30 \mu \mathrm{m}$ et d'épaisseur approximative entre 5 et $10 \mu \mathrm{m}$.

Les études isothermes sous atmosphère contrôlée d'argon ont été réalisées dans un four tubulaire.

Les analyses de diffraction $\mathrm{X}$ sur poudre à température ambiante et en température ont été effectuées respectivement au Centre de Diffractométrie de l'Université Claude Bernard Lyon1 et au Centre Spin de l'Ecole des Mines de Saint-Etienne avec la raie $\mathrm{K} \alpha \mathrm{l}$ du cuivre. 
Les images de Microscopie Electronique à Balayage ont été faites au Centre Technologique des Microstructures $(\mathrm{CT} \mu)$ de l'UCB Lyon1.

\section{RESULTATS ET DISCUSSION}

Une première étude générale par diffraction des rayons $X$ en température, sous atmosphère contrôlée, a été réalisée pour identifier les conditions de température nécessaires à la dismutation et les différentes phases formées lors de cette dismutation.

La figure ci-dessous (Fig. 1) montre l'évolution des phases présentes au cours du traitement d'une poudre de $\mathrm{SnO}$ sous hélium à des températures de $25,430,450,470,550$ et $50^{\circ} \mathrm{C}$.

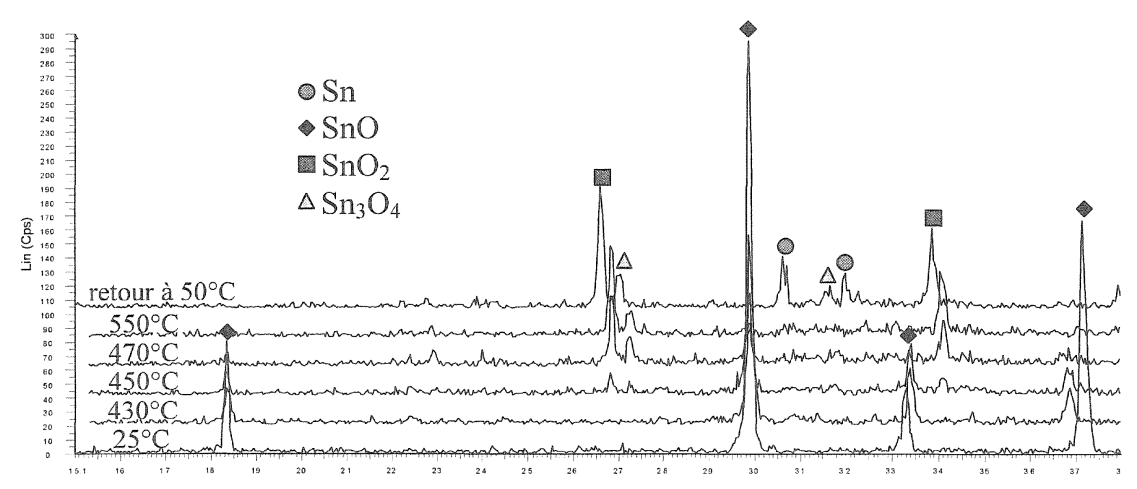

Figure 1 : Etude par diffraction $\mathrm{X}$ en température de la dismutation du monoxyde d'étain $\mathrm{SnO}$ (sous hélium)

Cette étude a montré, pour des températures comprises entre 430 et $550^{\circ} \mathrm{C}$, la disparition de la phase $\mathrm{SnO}$ et la formation de trois composés : $\mathrm{Sn}, \mathrm{SnO}_{2}$ et $\mathrm{Sn}_{3} \mathrm{O}_{4}$. L'étain est uniquement présent au retour à $50^{\circ} \mathrm{C}$, du fait de son passage à l'état liquide $\left(\mathrm{T}_{\mathrm{f}}=232^{\circ} \mathrm{C}\right)$.

Des analyses par diffraction des rayons $\mathrm{X}$, après refroidissement à température ambiante, ont ensuite été menées sur des poudres traitées en isotherme $\left(400\right.$ et $\left.480^{\circ} \mathrm{C}\right)$ sous argon afin de déterminer l'évolution qualitative et quantitative des phases formées ou disparues au cours du temps

La Fig. 2 montre l'évolution des diagrammes de diffraction X pour des angles $2 \theta$ compris entre 25 et $35^{\circ}$ et pour trois temps d'exposition de deux poudres de $\mathrm{SnO}$ soumises à 400 et $480^{\circ} \mathrm{C}$.

A $400^{\circ} \mathrm{C}$, après deux heures, la phase $\mathrm{SnO}$ est prépondérante alors que les phases $\mathrm{Sn}, \mathrm{SnO}_{2}$ et $\mathrm{Sn}_{3} \mathrm{O}_{4}$ commencent à apparaître. Après quatre heures, l'intensité du pic de $\mathrm{SnO}$ a considérablement diminué au profit des trois autres composés. Enfin, après seize heures de traitement, les pics de $\mathrm{SnO}$ ont disparu alors que les autres phases sont toujours présentes.

A $480^{\circ} \mathrm{C}$, après une heure, la phase Sno a déjà disparu et les phases $\mathrm{Sn}, \mathrm{SnO}_{2}$ et $\mathrm{Sn}_{3} \mathrm{O}_{4}$ sont présentes en quantité importante. $\mathrm{Au}$ cours du traitement, $\mathrm{Sn}_{3} \mathrm{O}_{4}$ tend ensuite à disparaître.

Une étude quantitative a été réalisée par dosage par rayons $\mathrm{X}$ en utilisant l'alumine $\alpha$ comme étalon interne. Malgré des incertitudes importantes, elle permet de noter l'évolution quantitative des espèces au cours des réactions. Les graphiques de la Fig. 3 donnent les pourcentages relatifs de $\mathrm{Sn}, \mathrm{SnO}$ et $\mathrm{SnO}_{2}$ pour les traitements à 400 et $480^{\circ} \mathrm{C}$ en fonction du temps. Les intensités des courbes de $\mathrm{Sn}_{3} \mathrm{O}_{4}$ sont données à titre indicatif, puisque ce composé n'a pu être isolé pour l'étalonnage interne du dosage. 

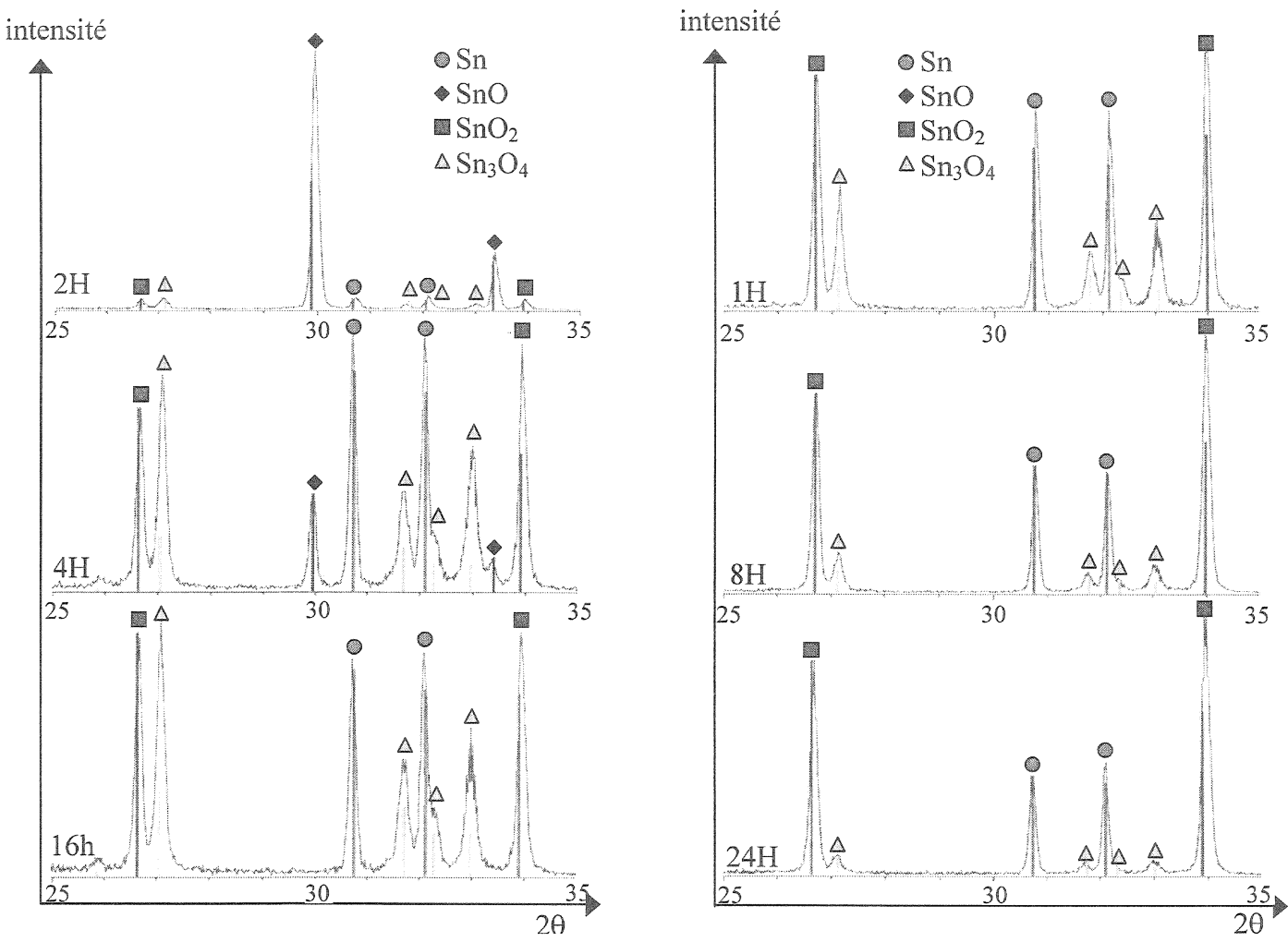

Figure 2 : Etudes par diffraction $\mathrm{X}$ de la dismutation de poudres de $\mathrm{SnO}$ traitées en isotherme à 400 et $480^{\circ} \mathrm{C}$
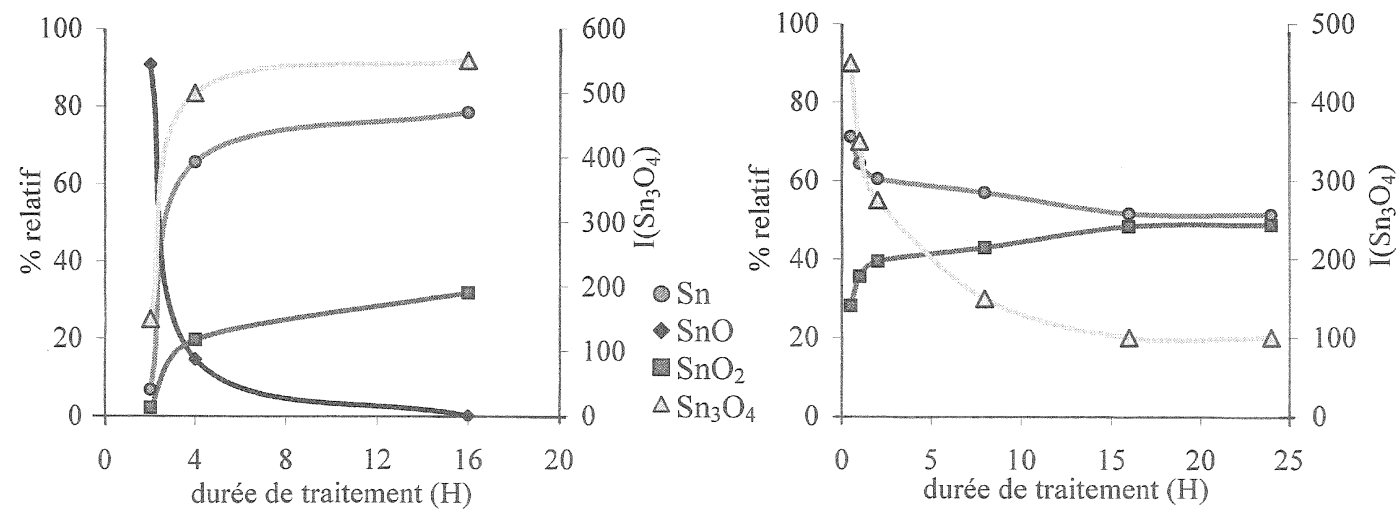

Figure 3 : Evolution quantitative des phases au cours de la dismutation à 400 et à $480^{\circ} \mathrm{C}$

Ces graphiques confirment les évolutions décrites précédemment. A $400^{\circ} \mathrm{C}$, les quantités de $\mathrm{Sn}, \mathrm{SnO}_{2}$ et $\mathrm{Sn}_{3} \mathrm{O}_{4}$ augmentent tandis que celle de $\mathrm{SnO}$ diminue jusqu'à s'annuler. $\mathrm{A} 480^{\circ} \mathrm{C}, \mathrm{SnO}$ a déjà disparu, la phase $\mathrm{Sn}_{3} \mathrm{O}_{4}$ décroît au cours du traitement, tandis que les quantités des composés $\mathrm{Sn}$ et $\mathrm{SnO}_{2}$ tendent à s'égaliser. 
Les évolutions données dans ces deux expériences s'expliquent par les réactions suivantes :

$$
\begin{aligned}
& 4 \mathrm{SnO} \rightarrow \mathrm{Sn}+\mathrm{Sn}_{3} \mathrm{O}_{4} \\
& \mathrm{Sn}_{3} \mathrm{O}_{4} \rightarrow \mathrm{Sn}+2 \mathrm{SnO}_{2}
\end{aligned}
$$

En effet, dans un premier temps, $\mathrm{SnO}$ disparaît au profit de $\mathrm{Sn}$ et $\mathrm{Sn}_{3} \mathrm{O}_{4}$ qui lui-même réagit pour former $\mathrm{Sn}$ et $\mathrm{SnO}_{2}$.

Ces deux réactions successives aboutissent à une réaction globale $2 \mathrm{SnO} \rightarrow \mathrm{Sn}+\mathrm{SnO}_{2}$.

Les pourcentages massiques théoriques de $\mathrm{Sn}$ et $\mathrm{SnO}_{2}$ après réaction sont respectivement de 44 et $56 \%$ en relatif. Ces deux valeurs confirment les pourcentages relatifs finaux en $\mathrm{Sn}$ et $\mathrm{SnO}_{2}$ obtenus après traitement de 24 heures à $480^{\circ} \mathrm{C}$ (Fig. 3).

Une étude morphologique a ensuite été effectuée sur la dismutation de $\mathrm{SnO}$. La figure suivante (Fig. 4) montre des photos de Microscopie Electronique à Balayage de l'évolution d'une poudre de $\mathrm{SnO}$ au cours de sa dismutation.

Les poudres obtenues en cours de dismutation et après dismutation totale montrent une hétérogénéité due au mélange des phases présentes: $\mathrm{Sn}, \mathrm{Sn}_{3} \mathrm{O}_{4}$ et $\mathrm{SnO}_{2}$ ou $\mathrm{Sn}$ et $\mathrm{SnO}_{2}$. Les grains sphériques sont probablement de l'étain formé à l'état liquide puis solidifié lors du refroidissement.

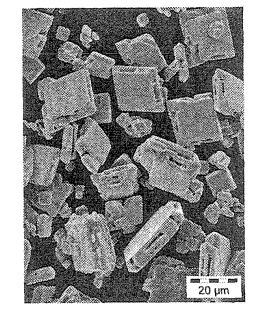

avant dismutation $\mathrm{SnO}$

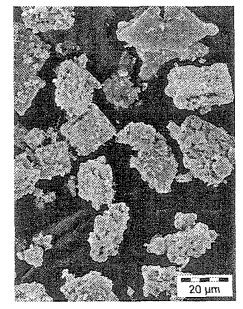

Isotherme $480^{\circ} \mathrm{C}$, 3heures $\mathrm{Sn}+\mathrm{Sn}_{3} \mathrm{O}_{4}+\mathrm{SnO}_{2}$
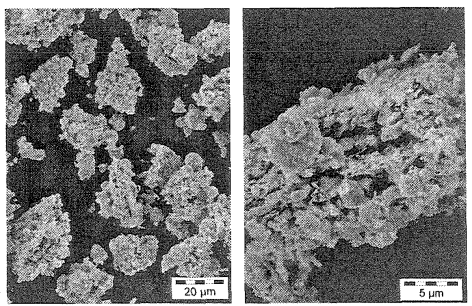

Isotherme $560^{\circ} \mathrm{C}, 3$ heures $\mathrm{Sn}+\mathrm{SnO}_{2}$

Figure 4 : Poudres de $\mathrm{SnO}$ avant dismutation, en cours de dismutation et après dismutation

\section{CONCLUSION}

La dismutation de $\mathrm{SnO}$ s'effectue en deux étapes simultanées. SnO se décompose tout d'abord assez rapidement en étain (liquide aux températures de traitement) et en $\mathrm{Sn}_{3} \mathrm{O}_{4}$, qui lui-même se décompose plus lentement en $\mathrm{SnO}_{2}$ et de nouveau en étain. La dismutation provoque également des changements morphologiques, dus au nombre important de phases présentes $\left(\mathrm{Sn}, \mathrm{SnO}_{2}, \mathrm{Sn}_{3} \mathrm{O}_{4}\right)$, et l'observation de billes solides qui résulteraient de l'expulsion d'étain liquide des grains de $\mathrm{SnO}$.

\section{Références}

1. J.F.Mcaleer, P.T.Moselay, J.O.W.Norris, D.E.Williams, Journal of the Chemical Society. Faraday Transactions I, 83, 1323 (1987).

2. I.T.Weber, R.Andrade, E.R.Leite, E.Longo, Sensors and Actuators B, 72, 180 (2001).

3. A.Marchand, O.Bucher, H.Delalu, G.Marichy, J.J.Counioux, 1991, French Patent n 9103550.

4. F.Gauzzi, B.Verdini, Inorganica Chimica Acta, 104, 1 (1985).

5. M.Sergio Moreno, G.Punte, G.Rigotti, R.C.Mercader, A.D.Weisz, Solid State Ionics, 144, 81 (2001). 